\title{
Magnetic resonance imaging findings in Axenfeld-Rieger syndrome
}

This article was published in the following Dove Press journal:

Clinical Ophthalmology

20 May 2013

Number of times this article has been viewed

\author{
Matthew T Whitehead ${ }^{1,2}$ \\ Asim F Choudhri ${ }^{1,2}$ \\ Sarwat Salim ${ }^{3}$ \\ 'Department of Radiology, University \\ of Tennessee Health Science Center, \\ Memphis, TN, USA; ${ }^{2}$ Le Bonheur \\ Neuroscience Institute, Le Bonheur \\ Children's Hospital, Memphis, TN, \\ USA; ${ }^{3}$ Department of Ophthalmology, \\ University of Tennessee Health \\ Science Center, Memphis, TN, USA
}

Correspondence: Sarwat Salim Associate Professor of Ophthalmology, Department of Ophthalmology, University of Tennessee Health Science Center, Hamilton Eye Institute, 930 Madison Ave, Memphis, TN 38103, USA

Tel $+90 \mid 4485492$

Fax+90 I28 75I 47

Emailssalim@uthsc.edu

\begin{abstract}
Axenfeld-Rieger syndrome (ARS) is a genetic disorder representing a disease spectrum resulting from neural crest cell maldevelopment. Glaucoma is a common complication from the incomplete formation of the iridocorneal angle structures. Neural crest cells also form structures of the forebrain and pituitary gland, dental papillae, aortic arch walls, genitalia, and long bones; therefore, patients with ARS manifest a wide range of systemic findings. To our knowledge, detailed magnetic resonance imaging findings have not been previously reported. We report a case of a 19-month-old Indian male diagnosed with ARS with emphasis on magnetic resonance imaging findings of the globes, brain, teeth, and skull base.
\end{abstract}

Keywords: ARS, glaucoma, megalocornea, orbit MRI

\section{Introduction}

Axenfeld-Rieger syndrome (ARS) is a rare developmental disorder with both ocular and systemic manifestations. It is transmitted by an autosomal dominant mode of inheritance with high penetrance and phenotypic variability. ${ }^{1,2} \mathrm{Up}$ to $50 \%$ of ARS cases may be related to genetic mutations involving one or both of two distinct genetic loci - pituitary homeobox 2 gene (PITX2) at 4q25, and forkhead box C1 gene (FOXC1) at $6 \mathrm{p} 25 .^{3-6}$ A $13 \mathrm{q} 14$ deletion has also been implicated, but the specific underlying genetic defect remains elusive. ${ }^{1}$ CYP1B1 and GJA1 mutations may also be causative in some ARS cases. ${ }^{7,8}$

PITX2 and FOXC1 encode transcription factors that orchestrate neural crest development by regulating the expression of target genes. Both PITX2 and FOXC1 are expressed and are interactive in the affected tissues in mouse ARS models. ${ }^{9}$ Notably, PITX2 can negatively regulate FOXC1. ${ }^{9}$ PITX2 mutations are mainly present in ARS with additional nonocular malformations. ${ }^{1} \mathrm{FOXC} 1$ mutations are primarily detectable in ARS without systemic malformations; however, some associated systemic cardiac and hearing abnormalities have been described. ${ }^{1}$ Although most PITX2 and FOXC1 mutations are point mutations, mutation types can be quite broad and heterogeneous, resulting in a wide range of clinical manifestations. ${ }^{1,40-13}$

Neural crest cells form large portions of the ocular anterior segment, including the iris stroma, cornea, and structures of the iridocorneal angle. ${ }^{14,15}$ In ARS, maldevelopment and failed regression of neural crests cells result in abnormal cell retention in the anterior segment of the eye. Contraction of this persistent abnormal primordial neural crest membrane over time causes pathologic changes in the iris, such as iris atrophy, corectopia, and ectropion uveae..$^{1,16,17}$ 
Abnormal cells that are present along the anterior chamber angle impede aqueous drainage through the trabecular meshwork and Schlemm canal. ${ }^{14}$ Consequently, glaucoma is an inherent risk of the disease process, developing in roughly $50 \%$ of patients. ${ }^{16}$ Glaucoma onset may not occur until childhood, early adulthood, or rarely, late adulthood; ${ }^{16}$ therefore, long-term clinical monitoring is necessary in these patients. In ARS patients that develop glaucoma, medical treatment with agents that decrease aqueous humor is usually initiated. When medical therapy fails, surgical options include either glaucoma filtration surgery or glaucoma drainage device insertion. ${ }^{1}$

Neural crest cells generate or contribute to the formation of a number of other systemic structures. Malformations of the sella turcica and pituitary gland, face, umbilical tissues, and teeth associated with ARS can be attributed to their neural crest origin. $2,3,13,18$

Abnormal sellar morphology, osseous "bridging" of the anterior and posterior clinoid processes, parasellar cysts, thickened dorsum sella, and steep clival angle are all previously described abnormalities associated with ARS. ${ }^{18}$ Since the normal development of the adenohypophysis requires normal neural crest cell differentiation, adenohypophyseal hypoplasia has also been described. ${ }^{16}$

Craniofacial abnormalities can include maxillary hypoplasia, telecanthus, thin lips, and external ear dysplasia. ${ }^{13,16}$ Umbilical anomalies are variable, including periumbilical skin redundancy, umbilical hernia, and omphalocele. ${ }^{13,19}$ Other previously reported anomalies in ARS include cardiac defects, meningiomas, sensorineural hearing loss, hypospadias, and hydrocephalus. ${ }^{19-21}$

Dental abnormalities in some patients with ARS include microdontia, hypodontia/oligodontia, abnormal teeth morphology, small roots, and taurodontia (increased pulp chamber-to-root ratio). Hypodontia occurs with an incidence of $5 \%-6 \%$ in the normal population. ${ }^{22}$ The overall incidence of taurodontism is roughly $0.3 \%$ in white Europeans. ${ }^{2}$

We report a case of a 19-month-old Indian male diagnosed with ARS with a special emphasis on magnetic resonance imaging (MRI) findings of the brain, teeth, and skull base. Although the clinical findings of ARS are well published in the ophthalmic literature, detailed MRI of ARS has not been previously well described.

\section{Case report}

An otherwise healthy 19-month-old Indian male was referred to the ophthalmology service for evaluation of congenital glaucoma as a result of bilateral megalocornea. The patient was a product of an uneventful pregnancy and delivery, born at term to first cousins.

On physical examination, intraocular pressures were measured by a Perkins tonometer (Haag Streit, Mason, Ohio, USA) at $12 \mathrm{mmHg}$ and $14 \mathrm{mmHg}$ in the right (OD) and left eye (OS), respectively. Megalocorneas were confirmed (16 mm, oculi uterque [OU]) (Figure 1). Posterior embryotoxon (Figure 2), ectropion uveae, and iris atrophy were present in both eyes. On gonioscopy, the angle structures were open to ciliary body band with some scattered peripheral anterior synechiae. Increased axial globe length was present bilaterally $(24.1 \mathrm{~mm}$ OD; $24.3 \mathrm{~mm}$ OS). On fundoscopy, increased cup-to-disk ratios were present (Figure 3). These clinical findings were consistent with a diagnosis of ARS. A comprehensive systemic evaluation demonstrated no additional abnormalities.

MRI was requested and performed to further evaluate the globes, intraorbital contents, and brain for additional imaging manifestations of ARS. Magnetic resonance (MR) of the orbits and brain with and without intravenous contrast material $(0.1 \mathrm{mmol} / \mathrm{kg}$ gadopentetate dimeglumine) was performed on a GE Signa HDxt 3.0T (General Electric Medical System, Waukesha, Wisconsin, USA). Multiplanar pulse sequences were obtained of the whole brain including fast spoiled gradient echo (FSPGR) brain volume (BRAVO), diffusion weighted and diffusion tensor images (DWI/DTI), T2-weighted (T2WI), T2/fluid attenuation inversion recovery (T2/FLAIR), T1/fluid attenuation inversion recovery (T1/FLAIR), coronal short tau inversion recovery (STIR), and postcontrast T1/FLAIR. Also, thin section images were obtained through the orbits including axial and coronal fat saturated (fat sat) T2WI, fat sat FSPGR, and postcontrast fat sat FSPGR. Transverse corneal diameters (limbus to limbus), anteroposterior globe diameter (cornea to optic nerve head), and anterior segment depth (superficial mid corneal surface to superficial mid lens surface) were measured using electronic calipers provided on the picture archiving and communications system on thin section axial T2WI. The clival angle was measured at the junction of a line drawn between the superior margin of the cribriform plate and dorsum sella, and a line drawn along the posterior margin of the clivus. The teeth

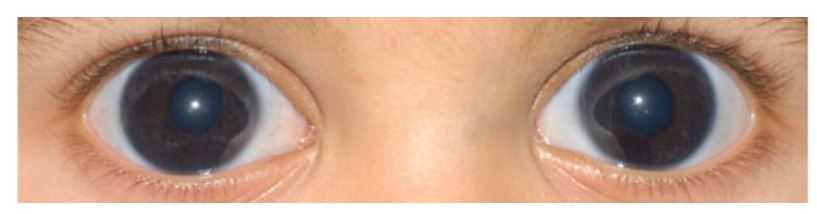

Figure I External photograph of the eyes showing megalocornea, measured at $16 \mathrm{~mm} \mathrm{OU}$.

Abbreviation: $\mathrm{OU}$, oculi uterque. 


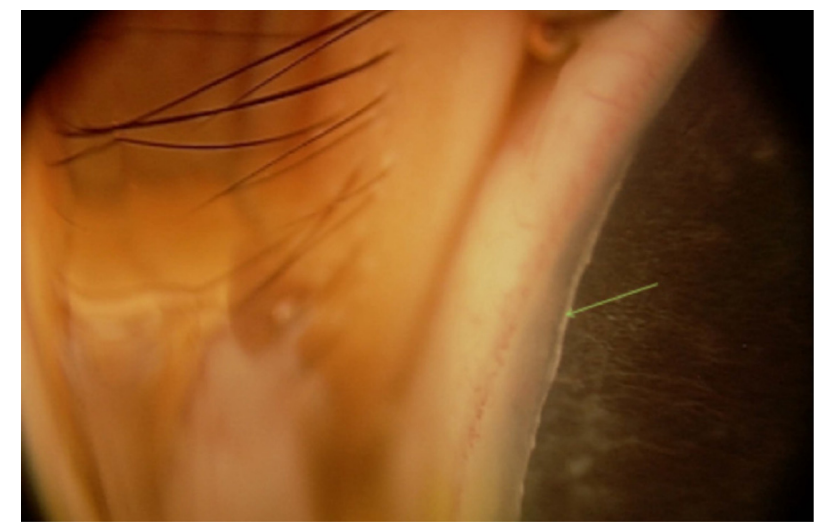

Figure 2 Anterior segment photograph showing posterior embryotoxon, an anteriorly displaced Schwalbe's line.

Note: The arrow indicates an anteriorly displaced Schwalbe's line.

were examined in all planes for dysmorphology; abnormal appearing pulp chambers were measured in the craniocaudal dimension on sagittal T1 FSPGR BRAVO images.

Orbit MR demonstrated lateralization and flattening of the limbus representing megalocornea (Figure 4). Thin section T2WI showed bilateral iris thinning as subjectively judged by two neuroradiologists (MW and AC) (Figure 4). Corneal diameters measured $15.2 \mathrm{~mm}(\mathrm{OD})$ and $15.1 \mathrm{~mm}$ (OS). Increased axial length (24.2 $\mathrm{mm}$ OD and $24.6 \mathrm{~mm}$ OS), enlarged anterior segments (3.6 $\mathrm{mm}$ anteroposterior dimension, $\mathrm{OU})$, lens rounding, bilateral optic nerve excavation, and optic nerve sheath prominence were also present (Figures 4 and 6), as compared to a normal orbital MRI in an aged-matched patient (Figure 5). The bilateral optic nerve intraorbital segments were normal.

There was a suggestion of taurodontia on MRI with teeth letters " $\mathrm{K}$ " and " $\mathrm{T}$ " displaying large pulp chamber-to-root ratios (pulp chambers measured $4.5 \mathrm{~mm}$ in the central craniocaudal dimension) (Figure 7).

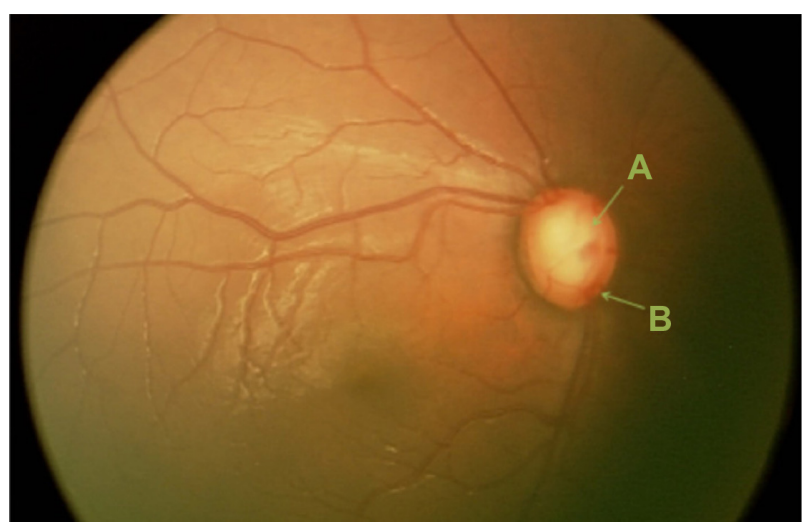

Figure 3 Fundus photograph of the right optic nerve depicting an enlarged cup-todisk ratio. (A) Cup; (B) disk.

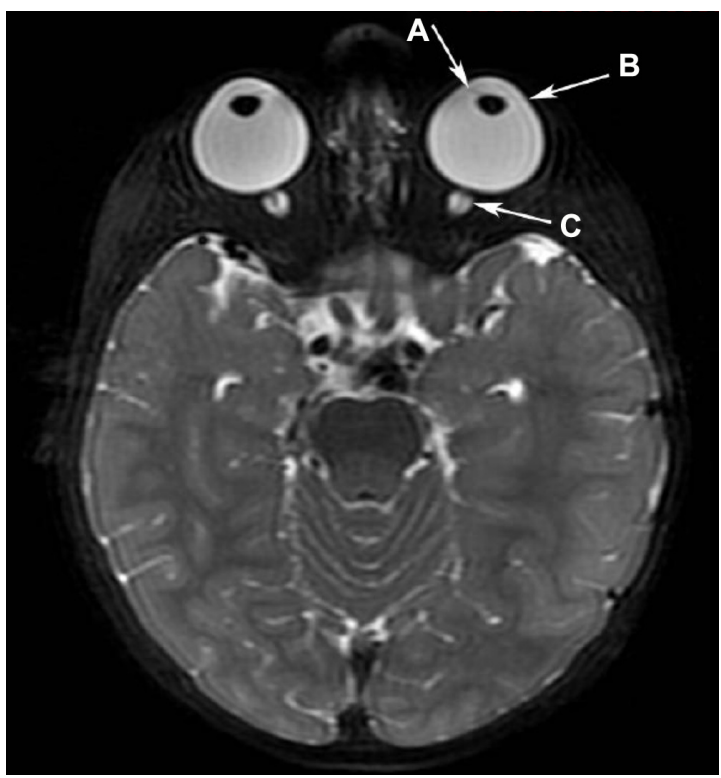

Figure 4 Axial T2WI depicting iris thinning in association with buphthalmos, enlarged anterior chambers, and lens rounding. (A) Iris thinning in association with buphthalmos, enlarged anterior chambers, and lens rounding. Megalocornea is present with (B) lateralization and flattening of the limbus. (C) The optic nerve sheaths are prominent.

Abbreviation: T2WI, T2-weighted image.

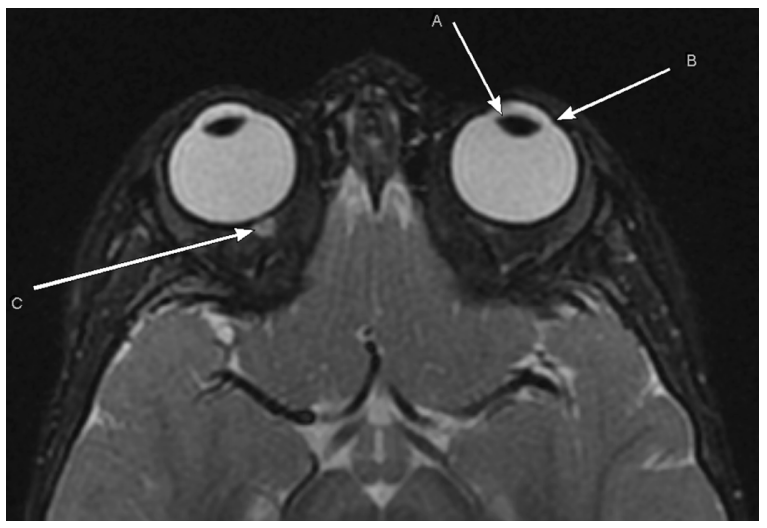

Figure 5 Axial $\mathrm{T} 2 \mathrm{Wl}$ from a normal orbital $\mathrm{MR}$ in an aged-matched patient demonstrating a normal oval lens morphology, the normal angle of the limbus, and a normal optic nerve-sheath complex. (A) Normal oval lens morphology; (B) normal angle of the limbus; (C) normal optic nerve-sheath complex.

Note: The normal lens is not well depicted in this section.

Abbreviations: T2WI, T2-weighted image; MR, magnetic resonance.

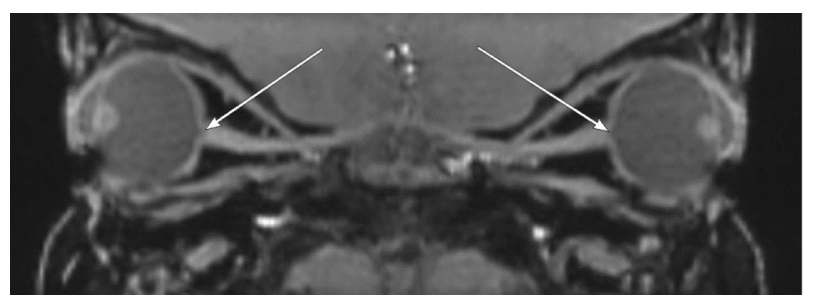

Figure 6 Sagittal reformation of thin section TI-weighted orbital images demonstrating enlarged cup-to-disk ratios bilaterally with optic nerve excavation.

Notes: Arrows depict enlarged bilateral cup-to-disk ratios with optic nerve excavation. Buphthalmos, deep anterior chambers, and lens rounding are also depicted. 

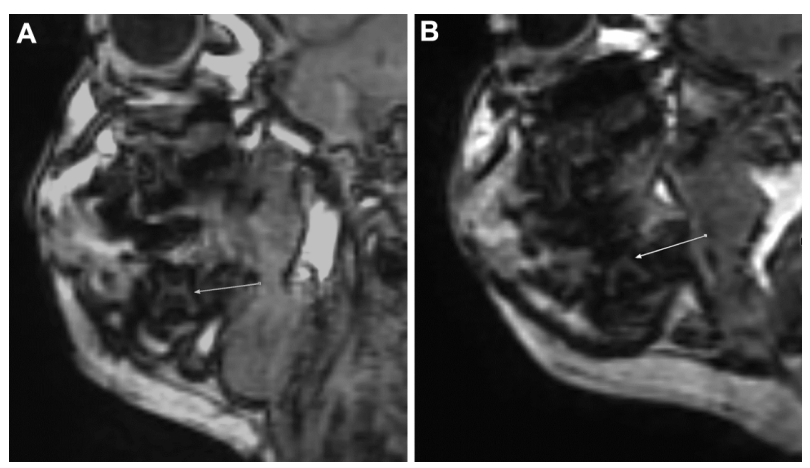

Figure 7 Sagittal FSPGR BRAVO image through the right mandible of our patient and a normal child. (A) Sagittal FSPGR BRAVO image through the right mandible of our patient showing evidence of a second deciduous mandibular molar taurodontia manifested by enlarged pulp chamber-to-root ratio (arrow). (B) Sagittal FSPGR BRAVO image through the right mandible of a 19-month-old normal child showing a normal second deciduous mandibular molar for comparison (arrow).

Abbreviation: FSPGR BRAVO, fast spoiled gradient echo brain volume.

Brain MR demonstrated a $4 \mathrm{~mm}$ uncomplicated pineal cyst and a $4 \mathrm{~mm}$ pars intermedia cyst associated with an otherwise normal pituitary gland (Figure 8). There was no evidence of osseous bridging of the sella turcica. The clivus was somewhat steep, with a clival angle of 111 degrees (Figure 8). Mild brachycephaly was also evident (Figure 8). Several small deep/paraventricular cerebral white matter lesions were present in both cerebral hemispheres, most conspicuous in the left frontal lobe. The left transverse sinus was aplastic. The dominant right transverse sinus received

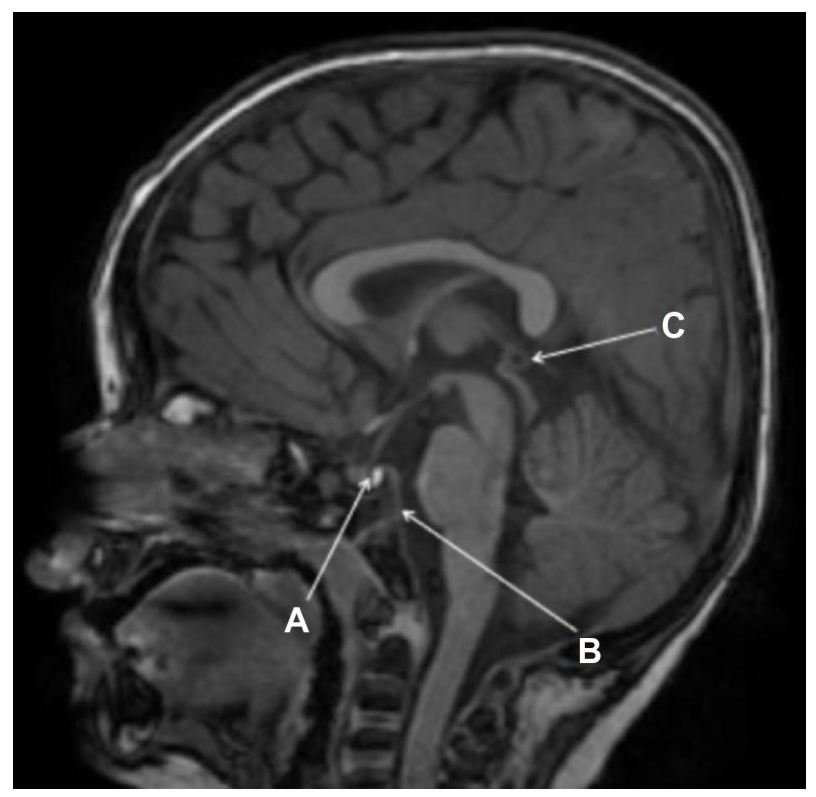

Figure 8 Sagittal FSPGR BRAVO image displaying a $4 \mathrm{~mm}$ pars intermedia pituitary cyst within an otherwise normal pituitary gland, a relatively steep clival angle, and a $4 \mathrm{~mm}$ pineal cyst is present. (A) A $4 \mathrm{~mm}$ pars intermedia pituitary cyst within an otherwise normal pituitary gland. (B) There is a relatively steep clival angle. (C) A $4 \mathrm{~mm}$ pineal cyst is present. Also note mild brachycephaly.

Abbreviation: FSPGR BRAVO, fast spoiled gradient echo brain volume. flow from both the sagittal sinus and straight sinus. A normal caliber, tortuous basilar artery was present, extending into the left cerebellopontine angle cistern. Normal caliber posterior communicating arteries were present. Mild symmetric T2 prolongation was seen in the central tegmental tracts without corresponding reduced diffusion. The olfactory tracts, imaged cervical spine, developing paranasal sinuses, and membranous labyrinths were normal. No malformations of cortical development were detectable.

\section{Discussion}

ARS represents a spectrum of developmental disorders resulting from abnormal migration and differentiation of neural crest cells with both ocular and systemic manifestations. Secondary glaucoma is a common complication from an incomplete development of the trabecular meshwork and Schlemm canal, affecting the aqueous outflow facility. Neural crest cells also generate structures related to the forebrain and pituitary gland, dental papillae, walls of the aortic arches, genitalia, and long bones. Therefore, patients with ARS manifest a wide range of systemic findings, including facial and dental abnormalities, pituitary gland and sella anomalies with growth hormone deficiencies, redundant periumbilical skin, hearing loss, cardiac anomalies, and hypospadias.

ARS remains primarily a clinical diagnosis with characteristic ocular findings including posterior embryotoxon (Figure 2), peripheral anterior synechiae, iris atrophy, bilateral corectopia, and secondary glaucoma. These patients may present with microcornea or megalocornea, the latter being present in our patient (Figure 1). The posterior embryotoxon is a prominent, anteriorly displaced Schwalbe's line.

MR screening of all patients in whom there is a low clinical suspicion of ARS would be impractical; however, in patients who present with characteristic clinical signs of ARS including abnormalities of the iris, cornea, face, and/or systemic manifestations, MR screening would be beneficial. At initial presentation, MR screening with both a brain MR and orbit MR would aid in the diagnosis of ARS; both ocular and nonocular intracranial manifestations could be revealed. Early recognition of enlarged anterior segments on MRI would prompt a detailed ophthalmic exam, as this finding can be an imaging sign of glaucoma. The initial orbital MRI would then serve as a baseline MR exam for comparison on follow-up. We recommend a follow-up orbital MRI in 6-12 months or earlier if symptoms/ signs progress. Beyond that, follow-up orbital MR could be performed at the discretion of the referring ophthalmologist. 
For the initial brain MR, a noncontrast study with basic T1WI, T2WI, T2/FLAIR, and DWI pulse sequences of the whole brain and thin section T1WI through the sella turcica is recommended. For both the initial and follow-up orbital MRIs, thin-section axial and coronal fat sat T2WI and pre- and postcontrast axial three-dimensional (3D) fat sat T1WI images through the orbits are advised.

There are many types of MR pulse sequences with various technical parameters and imaging planes that can be used to evaluate the brain and orbits in these patients. At our institution, we use the following sequences to evaluate the brain: sagittal FSPGR BRAVO, axial DWI/DTI, axial T2WI, axial T2/FLAIR, axial T1/FLAIR, and coronal STIR. Dedicated orbital MR pulse sequences include axial and coronal fat sat T2WI, axial fat sat FSPGR, and postcontrast axial fat sat FSPGR. The FSPGR BRAVO is a 3D volumetric acquisition that captures thin section images with near isotropic or isotropic spatial resolution (equal resolution in the transaxial and longitudinal planes). This provides the radiologist with thin section data that can be easily reformatted into any other plane for interpretation. The FSPGR BRAVO sequence displays anatomy, especially brain parenchymal anatomy, in fine detail. T1/FLAIR images are useful to evaluate the myelination pattern in children (especially those under 2 years of age). Other standard brain pulse sequences (T2WI, STIR, DWI) in children are more useful to detect pathologic parenchymal lesions such as acute or chronic white matter injury in patients with ARS. Thin section T1W1 through the sella turcica would aid in the evaluation of the pituitary and sellar abnormalities that have been previously described in ARS; ${ }^{18}$ both the pituitary parenchyma and surrounding osseous sella are well evaluated on this particular pulse sequence. Thin section fat sat T2WI through the orbits show fluid/tissue interfaces in detail while eliminating the signal from the normal intraorbital fat. Globe volumes, anterior segment size, corneal diameter, iris thinning, lens rounding, optic disk cupping, and optic nerve sheath size can all be assessed on T2WI. The noncontrast fat sat FSPGR pulse sequence also eliminates the intraorbital fat signal and aids in detection of complicated (hemorrhagic or proteinaceous) intraorbital fluid in all compartments. Also, it is the sequence to which the postcontrast fat saturated FSPGR sequence is compared. Postcontrast FSPGR pulse sequences through the orbits depict the normally enhancing uveal tract and retina.

Notable ocular MRI findings in our patient included megalocornea (lateralization and flattening of the limbus) and iris atrophy (apparent thinning on thin section T2WI) (Figure 4). Furthermore, MRI findings of buphthalmos, enlarged anterior segments, lens rounding, bilateral optic disk cupping, and optic nerve sheath prominence in our patient may be related to the underlying disease and/or a secondary manifestation of developing glaucoma.

Although dental examination yielded no evidence of microdontia or hypodontia, MRI demonstrated findings suggestive of possible taurodontism in the unerupted temporary second mandibular molars, a characteristic dental anomaly associated with ARS (Figure 7). However, the roots of these molars had not yet fully developed, and no radiographic images were available to validate these findings.

Brain MR abnormalities include small pars intermedia and pineal cysts, which could also be incidental findings (Figure 8). The sella turcica and pituitary gland were normal in morphology. A relatively steep clival angle was present as has been previously described in association with ARS (Figure 8). ${ }^{18}$ Signal alteration in the central tegmental tracts is probably unrelated and incidental, representing physiological maturation in a patient of this age..$^{23}$ The paraventricular cerebral white matter lesions were most likely sequelae of old nonspecific insults (Figure 8). We query whether these brain lesions in an otherwise healthy child and former term infant could be related to an underlying small arterial wall dysgenesis given the neural crest derivation of the muscular and connective tissue arterial walls of the ventral cephalic vascular tree. ${ }^{24}$ Mild vascular tortuosity of the basilar artery is an unusual finding in the general population in this age group; it is possible that this abnormality is related to dysgenesis of the arterial wall. However, the dorsal cephalic vascular tree is derived entirely from the mesoderm in quail-chick chimera. ${ }^{24}$ It is unclear if the left transverse sinus aplasia was an incidental finding or a product of the disease process.

In summary, MR imaging is a useful adjunct in the diagnosis and evaluation of patients with suspected ARS. Although MR lacks the sensitivity and specificity to substitute for a formal ophthalmic exam in the evaluation of suspected glaucoma, it may be used as an adjunctive tool to better delineate the anatomy of the globe and orbit. The change in appearance of any structure may be useful to monitor progression from an established baseline. Additionally, a brain MRI should be ordered at the time of presentation in all ARS patients to examine the structural anatomy of the intracranial compartment. The sella turcica, pituitary gland, clivus, and membranous labyrinths all require detailed scrutiny as sellar bridging, adenohypophyseal hypoplasia, steep clival angle, and sensorineural hearing loss have all been previously described in ARS patients. ${ }^{18,20}$ The dura should be evaluated 
for meningiomas. Given that neural crest cells form a portion of the wall of the ventral cephalic arteries, white matter lesions representing prior insults, as present in our patient, should be sought and the intracranial vascular tree should be thoroughly assessed. All teeth present on the brain MRI should also be screened by the radiologist for taurodontia. Consideration should be given to dental radiographs after a comprehensive examination by the dentist.

\section{Conclusion}

ARS represents a spectrum of developmental disorders with specific correlative MRI findings. To our knowledge, detailed MR findings of the primary disease process have not been previously reported in ophthalmic literature. As ARS often manifests systemic abnormalities in addition to ocular manifestations, evaluation and treatment necessitate a multidisciplinary approach. Radiographic imaging facilitates detection of abnormalities related to the brain, dental papillae, and craniofacial bones, and should be more readily obtained to deliver comprehensive care to these patients.

\section{Disclosure}

The authors report no conflicts of interest in this work.

\section{References}

1. Tumer Z Bach-Holm D. Axenfeld-Rieger Syndrome and Spectrum of PITX2 and FOXC1 mutations. Eur J Hum Genet. 2009;17(12):1527-1539.

2. Dressler S, Meyer-Marcotty P, Weisschuh N, et al. Dental and craniofacial anomalies associated with Axenfeld-Rieger syndrome with PITX2 mutation. Case Rep Med. 2010;2010:621984.

3. Semina EV, Reiter R, Leysens NJ, et al. Cloning and characterization of a novel bicoid-related homeobox transcription factor gene, RIEG, involved in Rieger syndrome. Nat Genet. 1996;14(4):392-399.

4. Asai-Coakwell M, Backhouse C, Casey RJ, Gage PJ, Lehmann OJ. Reduced human and murine corneal thickness in an Axenfeld-Rieger syndrome subtype. Invest Ophalmol Vis Sci. 2006;47(11):4905-4909.

5. Mears AJ, Jordan T, Mirzayans F, et al. Mutations of the forkhead/ winged-helix gene, FKHL7, in patients with Axenfeld-Rieger anomaly. Am J Hum Genet. 1998;63(5):1316-1328.

6. Nishimura DY, Swiderski RE, Alward WL, et al. The forkhead transcription factor gene FKHL7 is responsible for glaucoma phenotypes which map to 6p25. Nat Genet. 1998;19(2):140-147.

7. Tanwar M, Dada T, Dada R. Axenfeld-Rieger syndrome associated with congenital glaucoma and cytochrome P4501B1 gene mutations. Case Rep Med. 2010;2010:212656.

Clinical Ophthalmology

\section{Publish your work in this journal}

Clinical Ophthalmology is an international, peer-reviewed journal covering all subspecialties within ophthalmology. Key topics include: Optometry; Visual science; Pharmacology and drug therapy in eye diseases; Basic Sciences; Primary and Secondary eye care; Patient Safety and Quality of Care Improvements. This journal is indexed on Submit your manuscript here: http://www.dovepress.com/clinical-ophthalmology-journal
8. Cella W, de Vasconcellos JP, de Melos MB, et al. Structural assessment of PITX2, FOXC1, CYP1B1, and GJA1 genes in patients with AxenfeldRieger syndrome with developmental glaucoma. Invest Ophthalmol Vis Sci. 2006;47(5):1803-1809.

9. Berry FB, Lines MA, Oas JM, et al. Functional interactions between FOXC1 and PITX2 underlie the sensitivity to FOXC1 gene dose in Axenfeld-Rieger syndrome and anterior segment dysgenesis. Hum Mol Genet. 2006;15(6):905-919.

10. Footz T, Idrees F, Acharya M, Kozlowski K, Walter MA. Analysis of mutations of PITX2 transcription factor found in patients with AxenfeldRieger syndrome. Invest Ophthalmol Vis Sci. 2009;50(6):2599-2606.

11. Li D, Zhu Q, Lin H, Zhou N, Qi Y. A novel PITX2 mutation in a Chinese family with Axenfeld-Rieger syndrome. Mol Vis. 2008;14: 2205-2210.

12. Saadi I, Kuburas A, Engle JJ, Russo AF. Dominant negative dimerization of a mutant homeodomain protein in Axenfeld-Rieger syndrome. Mol Cell Biol. 2003;26(6):1968-1982.

13. Maciolek NL, Alward WL, Murray JC, Semina EV, McNally MT. Analysis of RNA splicing defects in PITX2 mutants supports a gene dosage model of Axenfeld-Rieger Syndrome. BMC Med Genet. 2006; $7: 59$.

14. Idrees F, Vaideanu D, Fraser SG, Sowden JC, Khaw PT. A review of anterior segment dysgeneses. Surv Ophthalmol. 2006;51(3):213-231.

15. Tripathi BJ, Tripathi RC. Neural crest origin of human trabecular meshwork and its implications for the pathogenesis of glaucoma. Am J Ophthalmol. 1989;107(6):583-590.

16. Shields MB. Axenfeld-Rieger syndrome: a theory of mechanism and distinctions from the iridocorneal endothelial syndrome. Trans Am Ophthalmol Soc. 1983;81:736-784.

17. Honkanen R, Alward WL, Cossari AJ. Progressive iris changes in a case of Axenfeld-Rieger syndrome. Arch Ophthalmol. 2006;124(12):1793.

18. Meyer-Marcotty P, Weisschuh N, Dressler P, Hartmann J, StellzigEisenhauer A. Morphology of the sella turcica in Axenfeld-Rieger syndrome with PITX2 mutation. J Oral Pathol Med. 2008;37(8): 504-510.

19. Jorgenson RJ, Levin LS, Cross HE, Yoder F, Kelly TE. The Rieger syndrome. Am J Med Genet. 1978;2(3):307-318.

20. Grosso S, Farnetani MA, Berardi R, et al. Familial Axenfeld-Rieger anomaly, cardiac malformations, and sensorineural hearing loss: a provisionally unique genetic syndrome? Am J Med Genet. 2002; 111(2):182-186.

21. Mammi I, De Giorgio P, Clementi M, Tenconi R. Cardiovascular anomaly in Rieger Syndrome: heterogeneity or contiguity? Acta Ophthalmol Scand. 1998;76(4):509-512.

22. Espinoza HM, Cox CJ, Semina EV, Amendt BA. A molecular basis for differential developmental anomalies in Axenfeld-Rieger syndrome. Hum Mol Genet. 2002;11(7):743-753.

23. Aguilera-Albesa S, Poretti A, Honnef D, et al. T2 hyperintense signal of the central tegmental tracts in children: disease or normal maturational process? Neuroradiology. 2012;54(8):863-871.

24. Etchevers HC, Vincent C, Le Douarin NM, Couly GF. The cephalic neural crest provides pericytes and smooth muscle cells to all blood vessels of the face and forebrain. Development. 2001;128(7):1059-1068.
PubMed Central and CAS, and is the official journal of The Society of Clinical Ophthalmology (SCO). The manuscript management system is completely online and includes a very quick and fair peer-review system, which is all easy to use. Visit http://www.dovepress.com/ testimonials.php to read real quotes from published authors. 